\title{
The mid-term periodicities in sunspot areas
}

\author{
Ryszarda Getko \\ Astronomical Institute, University of Wroclaw, Wroclaw, Poland \\ email: getko@astro.uni.wroc.pl
}

\begin{abstract}
The sunspot area fluctuations for the northern and the southern hemispheres of the Sun over the epoch of 12 cycles (12-23) are investigated. Because of the asymmetry of their probability distributions, the positive and the negative fluctuations are considered separately. The auto-correlation analysis of them shows three quasi-periodicities at 10, 17 and 23 solar rotations. The wavelets gives the 10-rotation quasi-periodicity. For the original and the negative fluctuations the correlation coefficient between the wavelet and the auto-correlation results is about 0.9 for $90 \%$ of the auto-correlation peaks. For the positive fluctuations it is also 0.9 for $70 \%$ of the peaks. For $90 \%$ of cycles in both hemispheres the auto-correlation analysis of negative fluctuations shows that two longer periods can be represented as the multiple of the shortest period. For positive fluctuations such dependences are found for more than $50 \%$ of cases.
\end{abstract}

Keywords. Sun: sunspots, methods: data analysis

\section{Introduction}

In the last decades, the intermediate quasi-periodicities of many solar activity tracers have been discussed. The about 12-rotation periodicity identified Krivova \& Solanki (2002) for sunspot data during 1749-2001. It was prominent during times of stronger activity, whereas it diminished and sometimes faded into the background during weak cycles. Getko (2006) found it in both high and low activity periods for the monthly Wolf numbers during cycles 1-22 and for the group sunspot numbers during cycles $5-22$. Two longer quasi-periodicities at 17 rotations and at 23 rotations were found in many solar activity parameters from the bottom of the convection zone to the atmosphere. More upto-date review is by Obridko and Shelting (2007). Here I present results from a statistical study of these periodicities in the sunspot areas during cycles 12-23. It enables one to deduce the mean length of the time period between strong fluctuations.

\section{A detailed analysis of quasi-periodicities}

I consider the daily sunspot areas for the northern hemisphere $\left(D_{l}^{n}\right)$, and the southern hemisphere $\left(D_{l}^{s}\right)$ for solar cycles $12-23$ available at the National Geophysical Data Center (NGDC) (http://solarscience.msfc.nasa.gov/greenwch/). For the $i$-th Carrington rotation I evaluate the mean sunspot area for the northern hemisphere $\left(S_{i}^{n}\right): S_{i}^{n}=\frac{1}{L} \sum_{l=1}^{L} D_{l}^{n}$, where $L$ is the number of days for the $i$-th rotation. I define the fluctuation $\left(F_{i}^{n}\right)$ of the mean sunspot area $\left(S_{i}^{n}\right)$ from the smoothed mean sunspot area: $F_{i}^{n}=S_{i}^{n}-\overline{S_{i}^{n}}$ for $i=$ $1, \ldots, N$, where $\overline{S_{i}^{n}}=\frac{1}{13} \sum_{j=i-6}^{i+6} S_{i}^{n}$. Each of the time series $\left\{F_{i}^{n}\right\}$ and $\left\{F_{i}^{s}\right\}$ contains $N=1706$ elements. Both have almost the same probability distributions. Fig. 1a shows the histogram of $\left\{F_{i}^{n}\right\}$ with a fitted Gaussian. The Kolmogorov-Lilliefors and ShapiroWilk tests reject the hypothesis of normality for them. Because each distribution has positive skew, the positive and the negative fluctuations are considered separately. For the northern hemisphere they can be defined as follows: 

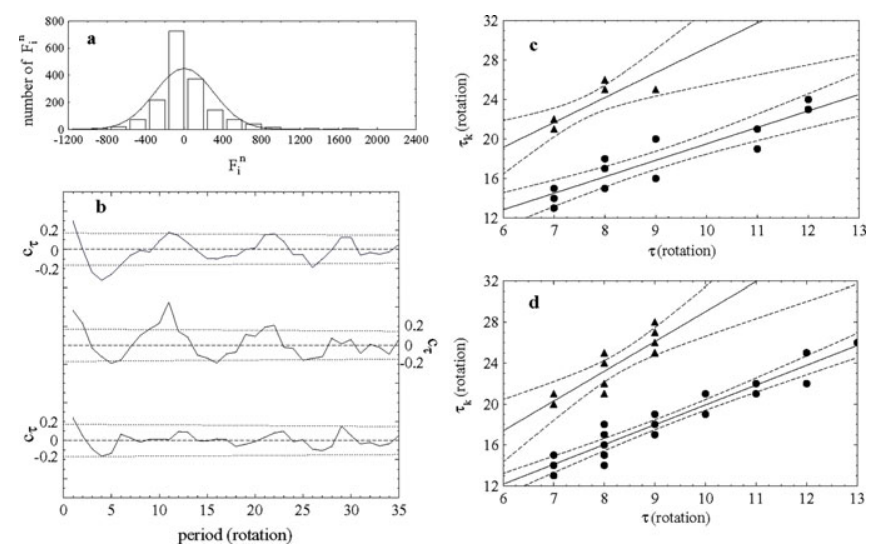

Figure 1. a Histogram of $\left\{F_{i}^{n}\right\}$ with a fitted Gaussian. b Top: Auto-correlation function $\left(c_{\tau}\right)$ of $\left\{F_{i}^{n}\right\}$ for cycle 18. Middle: Same as for the upper curve, but for $\left\{F_{i}^{n-}\right\}$. Bottom: Same as for the upper curve, but for $\left\{F_{i}^{n+}\right\}$. The dotted lines represent two standard errors of each $c_{\tau}$ function. c Dependence of $\tau_{2}$ and $\tau_{3}$ on $\tau$ for positive fluctuations. Lower solid curve represents the regression line for the points $\left(\tau, \tau_{2}\right)$ (dots). Upper solid curve represents the regression line for the points $\left(\tau, \tau_{3}\right)$ (triangles). Dashed lines represent the 95 per cent confidence interval for each regression line. $\mathbf{d}$ Same as for $\mathbf{c}$, but for negative fluctuations.

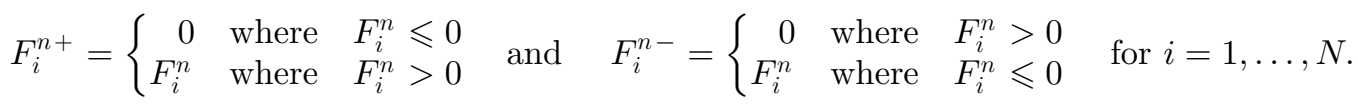

It is known that for a time series which contains Gaussian white noise and a sinusoidal component a probability distribution is symmetric and the auto-correlation functions $\left(c_{\tau}\right)$ of that time series, of its positive fluctuations and of its negative fluctuations should be the same. The functions $c_{\tau}$ of $\left\{F_{i}^{n}\right\},\left\{F_{i}^{n+}\right\}$ and $\left\{F_{i}^{n-}\right\}$ for one solar cycle are different (Fig. 1b). The functions $c_{\tau}$ of $\left\{F_{i}^{n}\right\}$ and $\left\{F_{i}^{n-}\right\}$ for cycle 18 have the significant global maxima at $\tau=11$ rotations and smaller maxima at $\tau_{1}=k * \tau$ for $k=2$ and 3. In $54 \%$ of 24 cases (12 cycles in each hemisphere) the functions $c_{\tau}$ of the original fluctuations have significant maxima for $\tau \in[7,13]$. In $30 \%$ cases the maxima for such $\tau$ belong to the interval $[1 \sigma, 2 \sigma]$. For positive fluctuations this contribution is $50 \%$ and $46 \%$ respectively. For negative fluctuations in $92 \%$ cases the maxima at $\tau \in[7,13]$ are significant. The mean value of all $\tau \in[7,13]$ for which the maxima are significant is approximately 10 rotations for all three fluctuation groups. I also consider the $c_{\tau}$ maxima for $\tau \in[14,19]$ and $\tau \in[20,27]$. For more than $50 \%$ of cases the positive fluctuations create the autocorrelation peaks for which the periods $\tau_{1} \approx 17$ and $\tau_{2} \approx 23$ can be represented as $\tau_{k} \approx k * \tau$ where $\tau \in[7,13]$ and $k=2$ or 3 . For each of $k$ the points $\left(\tau, \tau_{k}\right)$, the regression line (solid) and the $95 \%$ confidence interval for each line (dotted) are shown in Fig. 1c. For $k=2$ the correlation coefficient for 13 points is 0.91 , for $k=3$ it is 0.86 for 8 points. For the negative fluctuations the strong dependence between the considered periodicities was found in $\sim 90 \%$ for $k=2$ and in $\sim 50 \%$ for $k=3$ (Fig. 1d). For $k=2$ the correlation is 0.95 for 23 points and for $k=3$ it is 0.91 for 11 points. It is important to add that the $c_{\tau}$ values at $\tau>27$ are not reliable because of the solar cycle length.

I also applied the Morlet wavelet (Torrence \& Compo 1998) to three fluctuation time series for each of 24 cases. Fig. 2 shows the normalized wavelet maps for cycle 18 . Black contours denote the 95 per cent significance level for detected peaks. The wavelet map of $\left\{F_{i}^{n}\right\}$ (top) shows two significant peaks (at $\tau \approx 6$ and 8). They are mainly created by three strong fluctuations at the begining and at the end of the high activity period. During the remaining part of this period a rised power is at $\tau=11$ rotations. Moreover, 


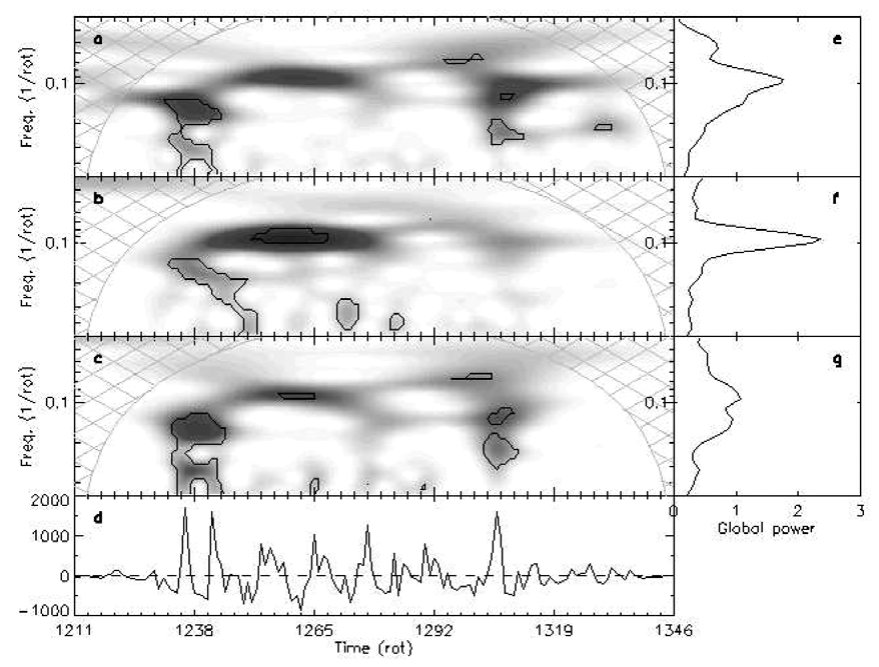

Figure 2. a-c: Wavelet power spectra of a: $\left\{F_{i}^{n}\right\}, \mathbf{b}:\left\{F_{i}^{n-}\right\}$ and $\mathbf{c}:\left\{F_{i}^{n+}\right\}$ mapping a time-frequency evolution of about 10-rotation periodicity. Top values of wavelet power are denoted by gradual darkening. Black contours denote significance levels of 95 per cent for detected peaks. A cone of influence is marked by the dashed region. e-g: Corresponding global wavelet power spectra. d: Time series $\left\{F_{i}^{n}\right\}$ for cycle 18 .

the integrated spectrum (right) shows the maximum at $\tau=11$ and confirms the autocorrelation results. For $\left\{F_{i}^{n-}\right\}$ (middle) the peak at $\tau=11$ is well detected with 95 per cent level, extends in time during the high activity period and dominates the integrated spectrum. The map of $\left\{F_{i}^{n+}\right\}$ (bottom) is similar to the map of $\left\{F_{i}^{n}\right\}$, but the peak at $\tau=11$ is significant. The global spectrum shows two almost the same peaks at $\tau=11$ and 8. Such an analysis was done for all 24 cases. The auto-correlation and the wavelet results are similar for $\tau \in[7,13]$ (the correlation between them is 0.9 for $87 \%, 92 \%$ and $72 \%$ of the auto-correlation peaks of $\left\{F_{i}^{n}\right\},\left\{F_{i}^{n-}\right\}$ and $\left\{F_{i}^{n+}\right\}$ respectively).

These results could indicate that the 10-rotation quasi-period is dominant. Getko (2004) showed that large activity complexes were responsible for strong sunspot number fluctuations. Thus, the time between strong fluctuations of toroidal magnetic flux in the tachocline could be on the order of 7-13 rotations. It is also well known that two solar hemispheres show certain hemispheric asymmetries in their solar-cycle features. However, two-sample Kolmogorov-Smirnov test shows that the 10-rotation quasi-periods evaluated for each of 12 solar cycles in each hemispheres do not differ. Two longer quasi-periods at about 17 and 23 rotations could be treated as subharmonics of the 10-rotation quasiperiod (Figs. 1c and 1d). This facts could explain a wide range of periodicities in various solar indices at all levels from the tachocline to the Earth.

\section{Conclusions}

(a) For both hemispheres the probability distributions of fluctuations are similar and have an asymmetry which means that there are more negative than positive fluctuations.

(b) The auto-correlation analysis of the original, the positive and the negative fluctuations prefers three quasi-periods: around 10, 17 and 23 rotations. The wavelet maps show one dominant quasi-period at about 10 rotations.

(c) For $90 \%$ of solar cycles in both hemispheres the auto-correlation analysis of negative fluctuations gives peaks for which the period $\tau_{2} \approx 17$ rotations can be represented 
as $\tau_{k} \approx k * \tau$ where $\tau \in[7,13]$ and $k=2$. For $k=3$ a such dependence are reliable in $50 \%$ of considered cases. For positive fluctuations such dependences are found for more than $50 \%$ of solar cycles in each hemispheres.

\section{References}

Getko, R. 2004, Solar Phys. 224, 291

Getko, R. 2006, Solar Phys. 238, 187

Krivova, N. A. \& Solanki, S. K. 2002, A $\&$ A 394, 701

Obridko, V. N. \& Shelting, B. D. 2007, Adv. Sp. Res. 40, 1006

Torrence, C. \& Compo, G. P. 1998, BAAS 79, 61 